\title{
PATRONES DE ASOCIACIÓN ENTRE VARIABLES DEL SUELO Y USOS DEL TERRENO EN LA CUENCA DEL RÍO LA VIEJA, COLOMBIA
}

\author{
Alexander Feijoo-Martínez, ${ }^{1}$ Maria C. ZúñIga, ${ }^{1}$ Heimar Quintero, ${ }^{2}$ Andrés \\ F. Carvajal-V anegas ${ }^{1}$ \& Diana P. Ortiz ${ }^{1}$ \\ ${ }^{1}$ Universidad Tecnológica de Pereira, Colombia. Vereda La Julita, Facultad de Ciencias Ambientales. \\ A.A. 097. E-Mail: afeijoo@utp.edu.co, connie@utp.edu.co, acarvaja@utp.edu.co,dportiz@utp.edu.co \\ ${ }^{2}$ Universidad Nacional de Colombia, Sede Palmira. A.A. 237.
}

Feijoo-Martínez, A., M. C. Zúñiga, H. Quintero, A. F. Carvajal-Vanegas \& D. P. Ortiz. 2010. Patrones de asociación entre variables del suelo y usos del terreno en la cuenca del río La Vieja, Colombia. Acta Zoológica Mexicana (n.s.), Número Especial 2: 151-164.

RESUMEN. En el Eje Cafetero de Colombia se diferenciaron patrones de uso del terreno con abundancia, diversidad y biomasa de invertebrados edáficos (coleópteros, diplópodos y lombrices de tierra), algunas variables físico-químicas e intensidad de uso. Los valores más elevados de abundancia y biomasa de coleópteros se encontraron en eucalipto-lulo (Solanum quitoense), con 48 ind. $\mathrm{m}^{-2}$ y $9.4 \mathrm{~g}$ peso fresco (g.p.f.) $\mathrm{m}^{-2}$, respectivamente. La abundancia y biomasa de diplópodos fue superior en relicto de selva (53 ind. $\mathrm{m}^{-2}$ y 4.8 g.p.f. $\mathrm{m}^{-2}$, respectivamente) y en cafetales variedad Colombia (39 ind. $\mathrm{m}^{-2}$ y 3.1 g.p.f. $\mathrm{m}^{-2}$, respectivamente). La abundancia de lombrices fue mayor en eucalipto-lulo (768 ind. $\mathrm{m}^{-2}$ ) y en cafetales variedad Colombia $\left(540\right.$ ind. $\mathrm{m}^{-2}$ ). Las variables biológicas y fisicoquímicas configuraron tres y cuatro patrones de usos del terreno, respectivamente. La coinercia sugirió tres patrones relacionados con humedad, $\mathrm{C}, \mathrm{N}$ y densidad aparente del suelo $(\mathrm{p}<0.02)$. En pastizales y cafetales variedad Colombia predominaron lombrices peregrinas (Pontoscolex corethrurus, Polypheretima elongata, Dichogaster annae, Perionyx excavatus) o coleópteros (Ataenius sp., Onthophagus atriglabrus, Dichotomius satanas) y los valores para $\mathrm{C}$ y $\mathrm{N}$ fueron bajos y la densidad aparente mayor. El análisis de la información diferenció coberturas amigables o críticas para la biodiversidad, lo cual es valioso en la planificación del territorio.

Palabras clave: Usos del terreno, análisis multivariado, propiedades fisicoquímicas, fauna del suelo, lombrices de tierra

Feijoo-Martínez, A., M. C. Zúñiga, H. Quintero, A. F. Carvajal-Vanegas \& D. P. Ortiz. 2010. Patterns of association between soil variables and land uses in the La Vieja River watershed, Colombia. Acta Zoológica Mexicana (n.s.), Número Especial 2: 151-164.

ABSTRACT. In the Coffee Belt of Colombia we studied different patterns in land use considering the interaction between abundance, diversity and biomass of soil invertebrates (millipedes, beetles and earthworms), some physicochemical variables and land use intensity. Highest abundance and biomass of Coleoptera were found in Eucalyptus-lulo (Solanum quitoense), with 48 ind. $\mathrm{m}^{-2}$ and $9.4 \mathrm{~g}$ fresh weight

Recibido: 16/05/2008; aceptado: 08/01/2010. 
(gfw) $\mathrm{m}^{-2}$, respectively. Millipede abundance and biomass was higher in the relict forest $\left(53 \mathrm{ind} . \mathrm{m}^{-2}\right.$ and $4.8 \mathrm{gfw} \mathrm{m}{ }^{-2}$, respectively) and in the coffee variety "Colombia" (39.4 ind. $\mathrm{m}^{-2}$ and $3.1 \mathrm{gfw} \mathrm{m}^{-2}$, respectively). Earthworm abundance was higher in Eucalyptus-lulo (768 ind. $\mathrm{m}^{-2}$ ) and in the coffee variety Colombia (540 ind. $\mathrm{m}^{-2}$ ). Biological and physicochemical variables permitted the differentiation of three and four land use patterns, respectively. When they were combined with coinertia analysis, three patterns were distinguished, related to soil moisture, $\mathrm{C}$ and $\mathrm{N}$ contents and bulk density $(\mathrm{p}<0.02)$. In pastures and the coffee variety Colombia, beetles (Ataenius sp., Onthophagus atriglabrus, Dichotomius satanas) and peregrine earthworms were dominant (Pontoscolex corethrurus, Polypheretima elongata, Dichogaster annae, Perionyx excavatus), soil $\mathrm{C}$ and $\mathrm{N}$ contents were low and bulk density higher. The analysis showed potential for separating biodiversity-friendly or biodiversity-critical areas and may be useful for land use planning in rural areas.

Keywords: Land use, multivariate analysis, physical and chemical properties, soil fauna, earthworms.

\section{INTRODUCCIÓN}

En las dos últimas décadas del siglo XX en el paisaje de la cuenca del río La Vieja (entre $4^{\circ} 04^{\prime}$ y $4^{\circ} 49^{\prime} \mathrm{N}$ y $75^{\circ} 24^{\prime}$ y $75^{\circ} 57^{\prime} \mathrm{O}$ ) el incremento en la superficie cafetera se hizo a expensas del pastizal, el huerto habitacional y los relictos de monte. Entre 1995 y 2004 los problemas fitosanitarios y la crisis de sobreoferta de café condujeron al aumento de las coberturas de pastos y cultivos de alimentos (Zúñiga et al. 2003, Zúñiga et al. 2004).

Con la agregación de los terrenos que cada agricultor asigna en la finca a determinada cobertura se pueden configurar patrones del paisajes en la vereda o en la microcuenca, los cuales son susceptibles de cartografiar o visualizar para describir las formas de las estructuras generadas, la dispersión de ellas, las consecuencias de las combinaciones en el tiempo y el espacio (Vincent et al. 1976).

La calificación de los mosaicos en el paisaje para determinar ambientes hostiles o amigables para la biodiversidad todavía no se ha adelantado para los agroecosistemas de las regiones neotropicales Andinas (Feijoo et al. 2006). El ejercicio requiere observaciones sistemáticas en la meso y macro escala espacial en el mediano y largo plazo de la forma como prosperan o desaparecen las organizaciones vivientes, nativas o introducidas, al transformarse las relaciones entre distribución y patrones (forma, orden, cualidad) de uso del terreno (Bennet et al. 2006) y estructura (sustancia, materia, cantidad), en concordancia con la teoría de sistemas (Capra 1998).

La perspectiva productivista utiliza más a menudo las propiedades físicas y químicas como indicadoras de la calidad del suelo. En la medida que se reconocen y valoran las funciones de conservación en las fincas se han ido haciendo visibles los papeles de los microorganismos e invertebrados en la fragmentación y descomposición de la materia orgánica del suelo y en la circulación de nutrimentos; además, se pueden asociar los cambios en diversidad y abundancia con el estrés que generan las prácticas agrícolas y las técnicas de cultivo (Parr et al. 1992, Blair et al. 1996).

Las lombrices de tierra, coleópteros y algunos diplópodos proveen servicios críticos al ecosistema (Lavelle et al. 1997), siendo las primeras altamente endémicas y su- 
jetas a competencia de especies pantropicales. Así mismo, con métodos integradores de procesos, dinámica y propiedades del suelo se pueden construir patrones, señales tempranas o indicadores de los procesos de degradación o de recuperación (Feijoo \& Knapp 1998a, Feijoo et al. 1998b, Feijoo et al. 1999, Lavelle et al. 2006).

La hipótesis propuesta en el presente trabajo consideró que la construcción de ambientes hostiles o amigables para la diversidad esta relacionada con las coberturas vegetales semi-naturales o cultivadas y con los itinerarios de prácticas o técnicas. También se supuso que la agrodiversidad se debe evaluar solamente después de definir el mosaico contextual y los códigos culturales que configuran los procesos de ocupación humana de los Andes de Colombia.

\section{MATERIAL Y MÉTODOS \\ Descripción del área de trabajo}

Los muestreos se realizaron durante cuatro semanas entre julio y agosto de 2005 en la cuenca del río La Vieja situada al sur occidente de Colombia en los municipios de Alcalá, Cartago, Circasia, Filandia y Quimbaya. Comprende zonas montañosas de la Cordillera Central entre 860 y 3200 m.s.n.m. (Fig. 1). Se diferencian el piso térmico cálido y medio y el clima es bimodal con dos temporadas secas (diciembre - febrero y junio - agosto) y dos lluviosas (marzo-mayo y septiembre-noviembre), con temperatura que van desde los 18 hasta $\operatorname{los} 22^{\circ} \mathrm{C}$ y precipitación promedia de $1300 \mathrm{~mm}$ anuales.

La cuenca tiene una superficie de aproximadamente $2925 \mathrm{~km}^{2}$ con densidad poblacional de 378 habitantes $\mathrm{km}^{2}$ (MAVDT et al. 2005). Se caracteriza por la presencia de Andosoles (Consociación Armenia), Inceptisoles (Asociación Alcalá - San Antonio) y Alfisoles (Asociación Modín Bretaña) en los subsistemas de transporte y dispersión de aguas (Fig. 1) y por mosaicos y parches diversificados de usos del terreno con predominio de pastizales (35.7\%), cafetales de variedad Colombia $(24.6 \%)$, cultivos semestrales y permanentes $(18.9 \%)$ de caña panelera, plátano, yuca, frutales, mezclas de cafetales con sombrío y árboles maderables, relictos de selva (16.5\%)aledaños a algunas quebradas y ríos o en áreas protegidas de algunas fincas y Guadua angustifolia (2\%).

\section{Selección de los usos del terreno}

En escala del paisaje se seleccionaron tres estratos con nueve ventanas en los subsistemas de transporte y dispersión de agua (Hamblin \& Christiansen 1998), en forma de malla de $1 \mathrm{~km}^{2}$. En cada ventana se situaron con el GPS, 16 puntos con una distancia entre si de 200 m (Fig. 1). El diseño utilizado, sistemático estratificado en dos dimensiones (modelo de red cuadrada) con selección de un par de números al azar, permitió fijar las coordenadas de la unidad superior izquierda, disminuir los efectos de la auto correlación (Cochran 1974) y construir la ventana alineada o desalineada de manera bidimensional de acuerdo con proyectos previos de otros programas internacionales (BIOASSESS 2003, Mathieu et al. 2005). 
Feijoo-Martínez et al.: Patrones de asociación

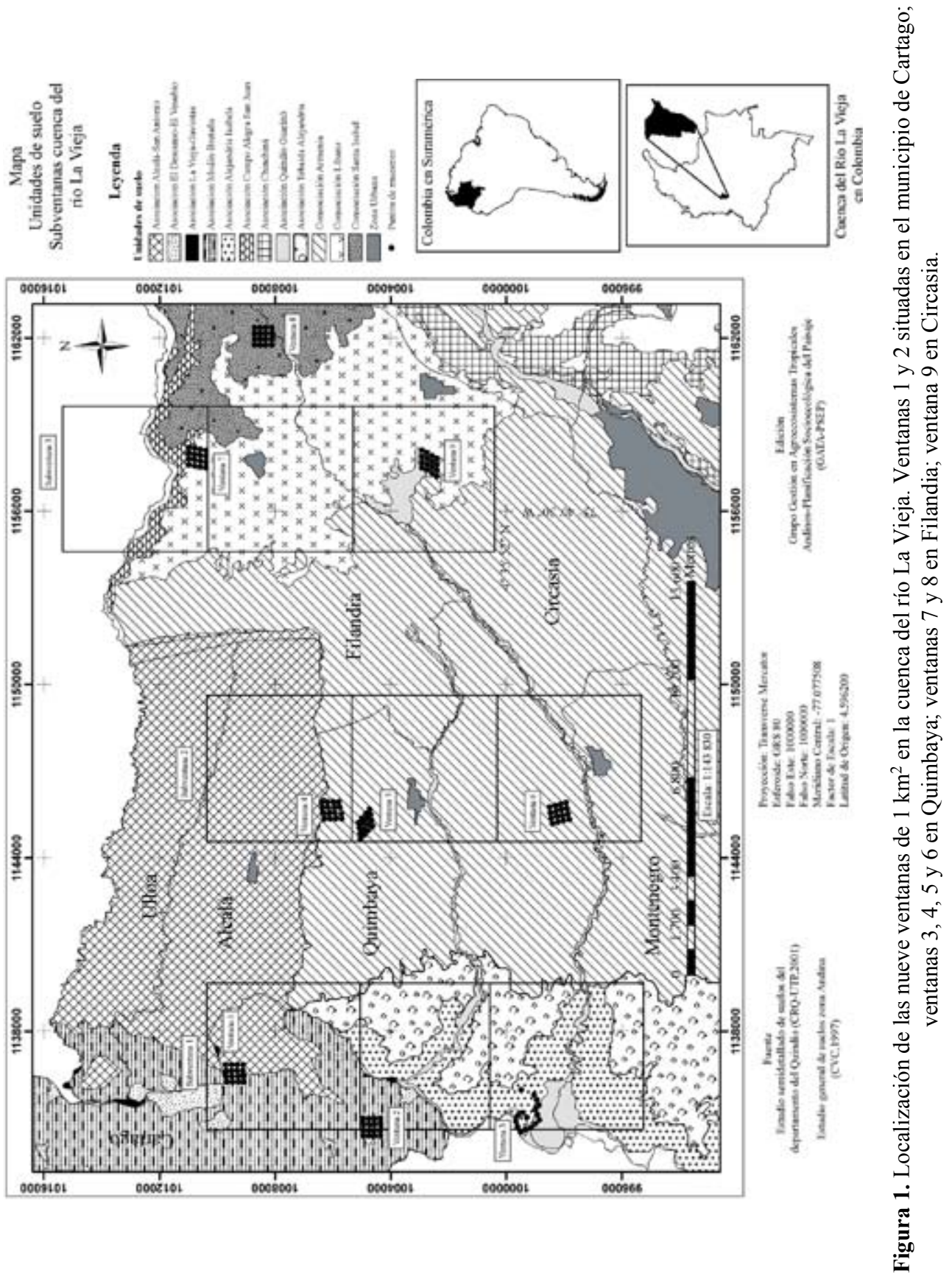




\section{Muestreo de las variables del suelo}

En cada punto de muestreo se tomó una muestra para densidad aparente (Blake \& Hartge 1986) y se cavó un monolito de suelo de $0.25 \times 0.25 \times 0.3 \mathrm{~m}$ de profundidad de acuerdo con el método del Programa Fertilidad Biológica de Suelos Tropicales (TSBF) (Anderson \& Ingram 1993) para muestrear, por cada estrato del suelo, las comunidades de lombrices de tierra, coleópteros, diplópodos. Además se extrajeron muestras de hojarasca superficial y suelo que se enviaron a la Universidad de Davis (EE.UU) para analizar el contenido de carbono y nitrógeno por el método de combustión seca a alta temperatura en un analizador CHN (Europa Scientific).

Los animales se identificaron hasta el nivel de familia, género o especie y se cuantificó la abundancia (individuos $\mathrm{m}^{-2}$ ), biomasa (gramos de peso fresco por $\mathrm{m}^{-2}$ ) y riqueza de especies (número de especies por uso de la tierra por ventana).

\section{Análisis de la información}

Para el análisis se definieron 13 usos del terreno y 12 variables biológicas, físicas y químicas (Cuadros I, II) de tres profundidades y la altura sobre el nivel del mar.

Cuadro I. Valores promedios de las características de los suelos en los 144 muestreos y 13 usos del terreno en la cuenca del río La Vieja, Colombia.

\begin{tabular}{|c|c|c|c|c|c|c|c|c|c|c|c|c|c|}
\hline \multirow[t]{2}{*}{ Uso del terreno } & \multirow[t]{2}{*}{$n$} & \multicolumn{2}{|c|}{$\begin{array}{l}\text { Altura sobre el } \\
\text { nivel del mar } \\
\text { (m) }\end{array}$} & \multicolumn{2}{|c|}{$\begin{array}{c}\text { Densidad } \\
\text { aparente } \\
\left(\mathrm{g} \cdot \mathrm{cm}^{-3}\right)\end{array}$} & \multicolumn{2}{|c|}{$\begin{array}{c}\text { Humedad } \\
\text { gravimétrica } \\
(\%)\end{array}$} & \multicolumn{2}{|c|}{$\begin{array}{l}\mathrm{C} \\
\%\end{array}$} & \multicolumn{2}{|c|}{$\begin{array}{l}\mathrm{N} \\
\%\end{array}$} & \multicolumn{2}{|c|}{ C:N } \\
\hline & & M & E. & M & E.E. & M & E. & M & $\mathrm{E}$ & M & E.E. & M & E.E. \\
\hline Ciprés & 7 & $1961^{b}$ & 55.3 & $0.6^{\mathrm{a}}$ & 0.08 & $38.3^{\mathrm{ab}}$ & 2.8 & $2.8^{\mathrm{b}}$ & 0.1 & $0.3^{\mathrm{bcd}}$ & 0.04 & $11.3^{\mathrm{c}}$ & $\overline{0.4}$ \\
\hline $\begin{array}{l}\text { Eucalipto } \\
\text { asociado con lulo }\end{array}$ & 2 & $1747^{\mathrm{ab}}$ & 0.0 & $0.7^{\mathrm{a}}$ & 0.05 & $37.5^{\mathrm{ab}}$ & 1.2 & $3.2^{\mathrm{b}}$ & 0.4 & $0.3^{\mathrm{d}}$ & 0.06 & $10.4^{\mathrm{abc}}$ & 0.2 \\
\hline Relicto de sc & 25 & $1685^{\mathrm{ab}}$ & 71.2 & $0.6^{\mathrm{a}}$ & 0.14 & $38^{\mathrm{ab}}$ & 2.5 & $2.9^{\mathrm{b}}$ & 0.1 & $0.3^{\mathrm{d}}$ & 0.08 & $10.5^{\mathrm{bc}}$ & 0.4 \\
\hline Barbecho & 9 & $1630^{\mathrm{ab}}$ & 134.1 & $0.7^{\mathrm{a}}$ & 0.17 & $38.9^{\mathrm{b}}$ & 2.5 & $2.6^{\mathrm{b}}$ & 0.2 & $0.3^{\mathrm{bcd}}$ & 0.08 & $11.2^{\mathrm{bc}}$ & 0.8 \\
\hline Cafe & 2 & $1544^{\mathrm{ab}}$ & 203.5 & $0.6^{\mathrm{a}}$ & 0.02 & $30.4^{\mathrm{ab}}$ & 5.3 & $2.3^{\mathrm{ab}}$ & 0.4 & $0.3^{\mathrm{d}}$ & 0.07 & $7.2^{\mathrm{ab}}$ & 2.6 \\
\hline Pastizal & 27 & $1484^{\mathrm{ab}}$ & 71.4 & $0.8^{\mathrm{a}}$ & 0.23 & $29.8^{\mathrm{ab}}$ & 2.3 & $1.8^{\mathrm{ab}}$ & 0.2 & $0.2^{\mathrm{abc}}$ & 0.12 & $13.3^{\mathrm{abc}}$ & 2.5 \\
\hline $\begin{array}{l}\text { Cafe } \\
\text { Colo }\end{array}$ & 13 & $1444^{\mathrm{ab}}$ & 58.4 & $0.7^{\mathrm{a}}$ & 0.06 & $32.8^{\mathrm{ab}}$ & 1.3 & $2.6^{\mathrm{b}}$ & 0.1 & $0.3^{\mathrm{d}}$ & 0.07 & $10.4^{\mathrm{abc}}$ & 0.5 \\
\hline Plátano & 7 & $1326^{\mathrm{a}}$ & 13.9 & $0.9^{\mathrm{a}}$ & 0.14 & $29.6^{\mathrm{ab}}$ & 2.0 & $2.2^{\mathrm{ab}}$ & 0.2 & $0.3^{\mathrm{cd}}$ & 0.06 & $8.4^{\mathrm{a}}$ & 0.4 \\
\hline Cafeta & 22 & $1316^{\mathrm{a}}$ & 2.5 & $0.8^{\mathrm{a}}$ & 0.11 & $30.9^{\mathrm{ab}}$ & 1.4 & $2.5^{\mathrm{b}}$ & 0.1 & $0.3^{\mathrm{d}}$ & 0.04 & $9.1^{\mathrm{ab}}$ & 0.3 \\
\hline Yuca & 2 & $1316^{\mathrm{ab}}$ & 3.5 & $0.9^{\mathrm{a}}$ & 0.12 & $28.1^{\mathrm{ab}}$ & 2.1 & $2.3^{\mathrm{ab}}$ & 0.2 & $0.2^{\mathrm{abcd}}$ & 0.07 & $10.3^{\mathrm{abc}}$ & 1.2 \\
\hline Guadual & 24 & $1152^{\mathrm{a}}$ & 33.1 & $0.9^{\mathrm{a}}$ & 0.21 & $24.2^{\mathrm{a}}$ & 1.8 & $1.5^{\mathrm{ab}}$ & 0.2 & $0.1^{\mathrm{ab}}$ & 0.11 & $15.6^{\mathrm{abc}}$ & 2.8 \\
\hline Cítricos & 1 & $1019^{a}$ & - & $0.9^{\mathrm{a}}$ & - & $18^{\mathrm{a}}$ & - & $0.9^{\mathrm{ab}}$ & - & $0.4^{\mathrm{d}}$ & - & $2.4^{\mathrm{a}}$ & - \\
\hline Caña panelera & 3 & $1002^{\mathrm{a}}$ & 4.3 & $0.9^{\mathrm{a}}$ & 0.29 & $22.7^{\mathrm{a}}$ & 6.0 & $0.4^{\mathrm{a}}$ & 0.2 & $0.1^{\mathrm{a}}$ & 0.04 & $10.6^{\mathrm{abc}}$ & 5.4 \\
\hline
\end{tabular}

$\mathrm{n}=$ número de muestreos en el uso; $\mathrm{M}=$ promedio de los muestreos por uso; E.E. $=$ error estándar; $\% \mathrm{C}=$ porcentaje de carbono orgánico; $\% \mathrm{~N}=$ porcentaje de nitrógeno orgánico; $\mathrm{C}: \mathrm{N}=$ relación carbono nitrógeno. Valores con letras diferentes presentaron diferencias altamente significativas $(p<0.05)$. 
Feijoo-Martínez et al.: Patrones de asociación

Cuadro II. Abundancia y biomasa de lombrices, coleópteros y diplópodos en los usos del terreno en la cuenca del río La Vieja, Colombia.

\begin{tabular}{|c|c|c|c|c|c|c|c|c|c|c|c|c|c|}
\hline \multirow{3}{*}{ Uso del terreno } & \multirow{3}{*}{$n$} & \multicolumn{4}{|c|}{ Lombrices } & \multicolumn{4}{|c|}{ Coleópteros } & \multicolumn{4}{|c|}{ Diplópodos } \\
\hline & & \multicolumn{2}{|c|}{$\begin{array}{l}\text { Abundancia } \\
\text { (ind. }^{-2} \text { ) }\end{array}$} & \multicolumn{2}{|c|}{$\begin{array}{l}\text { Biomasa } \\
\left(\text { g.p.f.m }{ }^{-2}\right)\end{array}$} & \multicolumn{2}{|c|}{$\begin{array}{l}\text { Abundancia } \\
\left(\text { ind. } \mathrm{m}^{-2} \text { ) }\right.\end{array}$} & \multicolumn{2}{|c|}{$\begin{array}{c}\text { Biomasa } \\
\text { (g.p.f.m }{ }^{-2} \text { ) }\end{array}$} & \multicolumn{2}{|c|}{$\begin{array}{l}\text { Abundancia } \\
\left(\text { ind. } \mathrm{m}^{-2} \text { ) }\right.\end{array}$} & \multicolumn{2}{|c|}{$\begin{array}{c}\text { Biomasa } \\
\text { (g.p.f.m }{ }^{-2} \text { ) }\end{array}$} \\
\hline & & M & E.E & $\mathrm{N}$ & $\mathrm{F}$ & M & E.E. & M & $\mathrm{F}$ & M & F E & M & E. \\
\hline iprés & 7 & 100.6 & 5 & $36 . j^{\circ}$ & 5 & $22.9^{\mathrm{a}}$ & 8.4 & $5.6^{\mathrm{a}}$ & & & 4.8 & $0.2^{\mathrm{a}}$ & 0.2 \\
\hline con lulo & 2 & $768^{\mathrm{b}}$ & 208 & $117.0^{\mathrm{a}}$ & 23.6 & $48.0^{\mathrm{a}}$ & 16.0 & $9.4^{\mathrm{a}}$ & 6 & $0.0^{\mathrm{b}}$ & 0.0 & $0.0^{\mathrm{b}}$ & 0.0 \\
\hline Relict & 25 & $183.7^{\mathrm{ab}}$ & 57.7 & $5^{\mathrm{a}}$ & 34.0 & $32.0^{\mathrm{a}}$ & 8.2 & $4.2^{\mathrm{a}}$ & 1.3 & 53 & 13.4 & $4.8^{\mathrm{a}}$ & 1.3 \\
\hline Barb & 9 & $330.7^{\mathrm{ab}}$ & 143.2 & $151.9^{\mathrm{a}}$ & 67.4 & $30.2^{\mathrm{a}}$ & 11.1 & $8.2^{\mathrm{a}}$ & 4.1 & $39.1^{\mathrm{a}}$ & 14.9 & $4.5^{\mathrm{a}}$ & 1.9 \\
\hline Cafe & 2 & $224.0^{\mathrm{ab}}$ & 96.0 & a & 26.2 & a & 8.0 & $0.2^{\mathrm{a}}$ & & & 0 & $0.0^{\mathrm{b}}$ & 0.0 \\
\hline astiz & 27 & $271.4^{\mathrm{ab}}$ & 41.9 & 10 & 26.9 & 30 & 9.7 & $4.6^{\mathrm{a}}$ & & $14.8^{\mathrm{a}}$ & & $1.2^{\mathrm{a}}$ & 0.7 \\
\hline dad & 13 & 540 & 9 & 1 & 25.2 & 22 & 6.2 & $3.1^{\mathrm{a}}$ & 1 & 39 & 17.8 & $3.1^{\mathrm{a}}$ & 1.8 \\
\hline látano & 7 & $326.9^{\mathrm{ab}}$ & 85.4 & $152.7^{\mathrm{a}}$ & 42.7 & $13.7^{\mathrm{a}}$ & 7.3 & $2.6^{\mathrm{a}}$ & & & & $3.4^{\mathrm{a}}$ & 3.1 \\
\hline Cafetal a & 22 & $268.4^{\mathrm{ab}}$ & 52.1 & $72.6^{\mathrm{a}}$ & 13.7 & $24.0^{\mathrm{a}}$ & 7.2 & $4.3^{\mathrm{a}}$ & 1.8 & $48.0^{\mathrm{a}}$ & 16.0 & $3.4^{\mathrm{a}}$ & 1.0 \\
\hline Yuca & 2 & $128.0^{\mathrm{a}}$ & 128.0 & $43.5^{\mathrm{b}}$ & 43.5 & $8.0^{\mathrm{a}}$ & 8.0 & $2.6^{\mathrm{a}}$ & 2.6 & $0.0^{\mathrm{b}}$ & .0 & $0.0^{\mathrm{b}}$ & 0.0 \\
\hline Guadual & 24 & $291.3^{\mathrm{ab}}$ & 60.4 & $117.6^{\mathrm{a}}$ & 26.0 & $20.7^{\mathrm{a}}$ & 6.0 & $3.9^{\mathrm{a}}$ & 1.5 & $30.7^{\mathrm{a}}$ & 10.5 & $5.2^{\mathrm{a}}$ & 2.7 \\
\hline Cítricos & 1 & $96.0^{\mathrm{ab}}$ & - & $11.7^{\mathrm{a}}$ & - & $0.0^{\mathrm{a}}$ & - & $0.0^{\mathrm{a}}$ & - & $0.0^{\mathrm{b}}$ & - & $0.0^{\mathrm{b}}$ & - \\
\hline Caña panelera & 3 & $154.7^{\mathrm{ab}}$ & 76.9 & $317.6^{\mathrm{a}}$ & 206.4 & $0.0^{\mathrm{a}}$ & 0.0 & $0.0^{\mathrm{a}}$ & 0.0 & $0.0^{\mathrm{b}}$ & 0.0 & $0.0^{\mathrm{b}}$ & 0.0 \\
\hline
\end{tabular}

Valores con letras diferentes presentaron diferencias altamente significativas $(p<0.05) . n=$ número de muestreos en el uso; $\mathrm{M}=$ promedio de los muestreos por uso; E.E. = error estándar.

Los cambios en las comunidades de lombrices, coleópteros y diplópodos permitieron describir la variación introducida por las coberturas y por el manejo de los agroecosistemas. Por medio de la densidad aparente se determinó el grado de compactación del suelo, mientras que el $\mathrm{C}, \mathrm{N}$ y la relación $\mathrm{C}: \mathrm{N}$ posibilitaron asociar la diversidad y abundancia de la edafofauna con la oferta y calidad de nutrientes en cada cobertura del terreno.

Para determinar si existían diferencias significativas por el uso del terreno en el comportamiento de las variables fisicoquímicas y biológicas (por separado) o correspondían a variaciones aleatorias (Siegel 1974), se aplicó el análisis de varianza no paramétrica para muestras independientes (prueba de Kruskal-Wallis). Posteriormente se agruparon los registros según variables biológicas y fisicoquímicas y se realizó un análisis de componentes principales (ACP) para reducir la dimensionalidad de la información de los datos con la construcción de combinaciones lineales de las variables originales que explicaban la varianza de la información, buscando reducirla de tal manera que se asumiera la mayor parte de la variabilidad. La influencia de las va- 
riables definidas en la clasificación de patrones, de acuerdo con el uso del terreno, se determinó con el análisis discriminante a partir del estimador de probabilidad de clasificación basado en la distancia de Mahalanobis entre dos grupos (Mclachlan 1992). El análisis de coinercia permitió establecer el nivel de probabilidad de la covariación entre los datos procedentes de registros biológicos y físicoquímicos (Velásquez et al. 2007), para lo cual se usaron los programas estadísticos ADE4 (Thioulouse et al. 1997) y SPSS (SPSS Inc. 1999).

\section{Coberturas vegetales de los terrenos}

\section{RESULTADOS}

En las ventanas 1 y 2 los usos con mayor número de muestreos fueron guadua (Guadua angustifolia) y pasto estrella (Cynodon nlemfuensis); en la 3 fueron los relictos de selva y guadua; en la 4 y 6 cafetal-plátano, en la 5 cafetal variedad Colombia; en la 7 pastizales y relictos de selva; en la 8 relictos de selva y ciprés; y en la 9 relicto de selva y cafetal con variedad Colombia (Fig. 2).

Las característica de los suelos de la cuenca del río La Vieja variaron significativamente $(\mathrm{p}<0.05)$ de acuerdo con la altura sobre el nivel del mar (entre $1002 \mathrm{~m}$ y $1961 \mathrm{~m})$ : los valores de C (\%) y N (\%) que oscilaron entre 3.2 y $0.3 \%$ en plantación de eucalipto asociado con lulo $(1747 \mathrm{~m})$ hasta 0.4 y $0.1 \%$, respectivamente, en la caña panelera (1002 m); la densidad aparente disminuyó con la altitud (de 0.9 a 0.6 g.cm ${ }^{-3}$ );

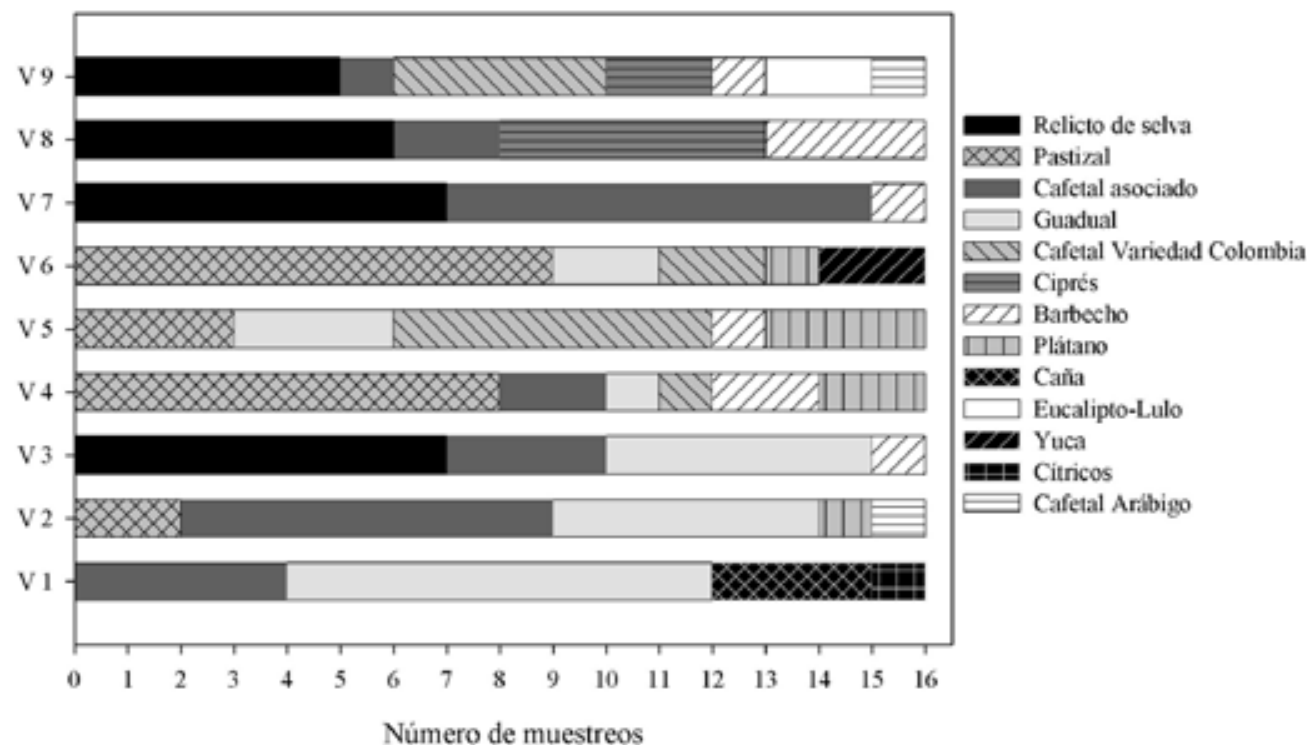

Figura 2. Frecuencia de coberturas vegetales en los muestreos realizados en las nueve ventanas (V1 a V9) de la cuenca del río La Vieja. 
la humedad más baja (18\%) ocurrió en cítricos (1019 m) y las más altas (38 a 39\%) en barbechos $(1630 \mathrm{~m})$ y relictos de selva $(1685 \mathrm{~m})$. La relación C:N varió desde 2.4 en los cítricos hasta 15.6 en los guaduales $(1152 \mathrm{~m})$ y no hubo efecto importante de la altitud (Cuadro I).

\section{Comunidades de macroinvertebrados en los usos del terreno}

Se colectaron 2538 lombrices agrupadas en 35 especies, 16 de las cuales se clasificaron en la familia Glossoscolecidae (Martiodrilus, Glossodrilus, Onychochaeta, Aptodrilus, Andiodrilus, Thamnodrilus, Periscolex y Pontoscolex), cinco en Megascolecidae (Amynthas, Polypheretima), tres en Acanthodrilidae (Dichogaster) y una en Lumbricidae; las 10 restantes se agruparon en desconocidas por no haber encontrado adultos o por presentar estructuras poco visibles, difusas o no comparables. Trece fueron nativas y siete exóticas, en las restantes no fue posible definirlo. Las especies se agruparon en las categorías ecológicas epígeas (25) y endógeas (10), la mayoría de ellas (14) habitan las capas $0-20 \mathrm{~cm}$, con distribución promedio vertical a $7.5 \mathrm{~cm}$ de profundidad.

En Coleoptera se encontraron 224 individuos de 62 especies entre las que se destacan Ataenius sp., Onthophagus atriglabrus y Dichotomius satanas. Se recolectaron varias especies de larvas de escarabajos depredadores como Selenophorus, Carabidae, Conoderus (Elateridae), Osorius y otros Staphylinidae, y varios grupos de Ostomidae (pos. Temnocheila sp.) y Cicindelidae (pos. Pseudoxychila bipustulata Latr) en los usos con relicto de selva, lo cual supone el ensamblaje de escarabajos depredadores y relaciones tróficas asociadas con coberturas arbóreas y aportes mayoritarios de hojarasca al suelo. En pastizales y cultivos permanentes se encontraron 38 géneros que indicaron la dinámica saprófaga.

Se identificaron 15 morfotipos de diplópodos situados en siete familias y tres órdenes. Polydesmida fue el orden más diverso con cinco morfotipos pertenecientes a cuatro familias; Spirostreptida registró cuatro morfotipos de dos familias; Siphonophoridae dos morfotipos y una familia; Spirobolidae y Glomeridesmidae registraron tres y un morfotipos respectivamente, pertenecientes a una sola familia cada uno.

La abundancia de lombrices fue mayor en el eucalipto maderable introducido de Australia, asociado con lulo $\left(768 \pm 208\right.$ ind. $\left.\mathrm{m}^{-2}\right)$ y en el cafetal a plena exposición y cultivo limpio de Variedad Colombia $\left(540.3 \pm 69.9\right.$ ind. $\left.\mathrm{m}^{-2}\right)$ con diferencias significativas $(\mathrm{p}<0.05)$, mientras que los números más bajos se encontraron en cítricos (96 ind. $\mathrm{m}^{-2}$ ) y ciprés $\left(100.6 \pm 44.5\right.$ ind. $\left.\mathrm{m}^{-2}\right)$. En la biomasa de lombrices los valores más altos fueron para caña panelera $\left(317.6 \pm 206.4\right.$ g.p.f.m $\left.{ }^{-2}\right)$ y el cultivo de plátano $\left(152.7 \pm 42.7\right.$ g.p.f. $\left.{ }^{-2}\right)$ y los más bajos se registraron en cítricos $\left(11.7\right.$ g.p.f.m $\left.{ }^{-2}\right)$.

En coleópteros los valores más elevados de abundancia y biomasa se encontraron en el eucalipto asociado con lulo ( $48 \pm 16$ ind. $\mathrm{m}^{-2} ; 9.4 \pm 6.1$ g.p.f. $\mathrm{m}^{-2}$ respectivamente), y los menores en cítricos y caña panelera. La abundancia y biomasa de diplópodos 
fue superior en el relicto de selva $\left(53.1 \pm 13.4\right.$ ind. $\mathrm{m}^{-2}$ y $4.8 \pm 1.3$ g.p.f.m ${ }^{-2}$, respectivamente) y en el cafetal asociado ( $48 \pm 16$ ind. $\mathrm{m}^{-2}$ y $3.4 \pm 1$ g.p.f.m $\left.{ }^{-2}\right)$ (Cuadro II).

\section{Patrones de asociación entre las variables del suelo y usos del terreno}

El ACP para las variables fisicoquímicas separó significativamente $(\mathrm{p}<0.01)$ cuatro grupos de uso del terreno. Los dos primeros componentes explicaron el $79 \%$ de variabilidad total y contrastó un primer grupo de usos con elevada humedad del suelo (CE, RS y BA) versus el segundo con suelos compactados y con signos de erosión (PL, $\mathrm{YU}, \mathrm{CI})$; el tercero grupo unió usos con alto valor de C (\%) y N (\%) (CAR, EL, CA, $\mathrm{CVC}$ ) opuestos al cuarto grupo con bajos valores (CÑ, PA y GU) (Fig. 3).

Los dos primeros componentes del ACP para las variables biológicas explicaron el $40.3 \%$ (23.3 y $17.2 \%)$ de la variabilidad total $(\mathrm{p}<0.01)$. En ellos se detectó tres grupos de usos del terreno: el primero grupo opuso usos del terreno con alta abundancia de lombrices, y baja de coleópteros y diplópodos ( $\mathrm{CVC}$ y EL) versus el segundo con bajos valores para los tres grupos (CI, YU, CÑ, CE), mientras que un tercer grupo diferenció usos con valores intermedios de abundancia y presencia de lombrices, coleópteros y diplópodos (RS, GU, CA, PL, BA, PA) (Fig. 4).
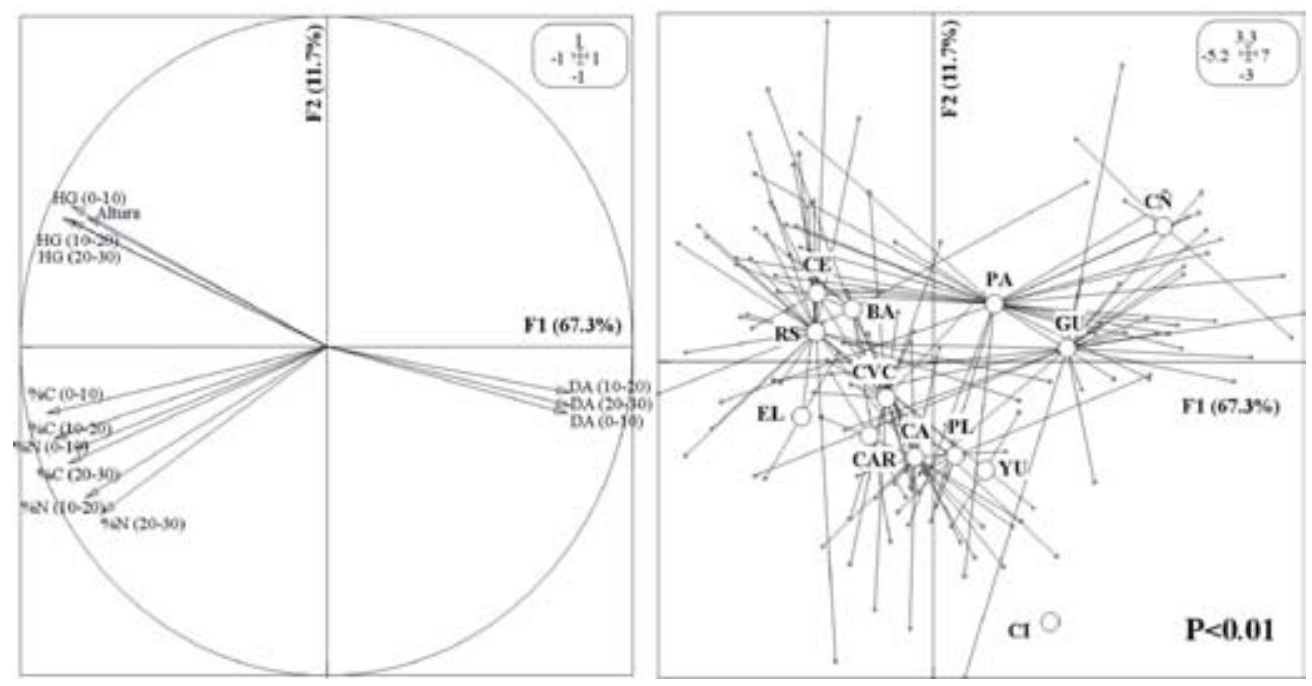

Figura 3. Proyección de los patrones asociados con la conservación y degradación de los usos del terreno en la cuenca del río de La Vieja, para las variables físicoquímicas (HG=Humedad gravimétrica, $\mathrm{DA}=$ Densidad aparente, Altura $=$ Altitud del local) en las diferentes profundidades del suelo (0-10, 10-20 y 20-30 cm), definidas por el ACP en el espacio factorial. Usos: $\mathrm{BA}=$ barbecho, $\mathrm{CA}=$ cafetal asociado, $\mathrm{CAR}=$ cafetal arábigo $\mathrm{CE}=$ Ciprés, $\mathrm{CVC}=$ cafetal variedad Colombia, $\mathrm{CN}=$ caña panelera, $\mathrm{CI}=$ cítricos, $\mathrm{EL}=$ eucalipto-lulo, $\mathrm{GU}=$ guadua, $\mathrm{PA}=$ pastizal, $\mathrm{PL}=$ plátano, $\mathrm{RS}=$ relicto de selva, $\mathrm{YU}=$ yuca. 

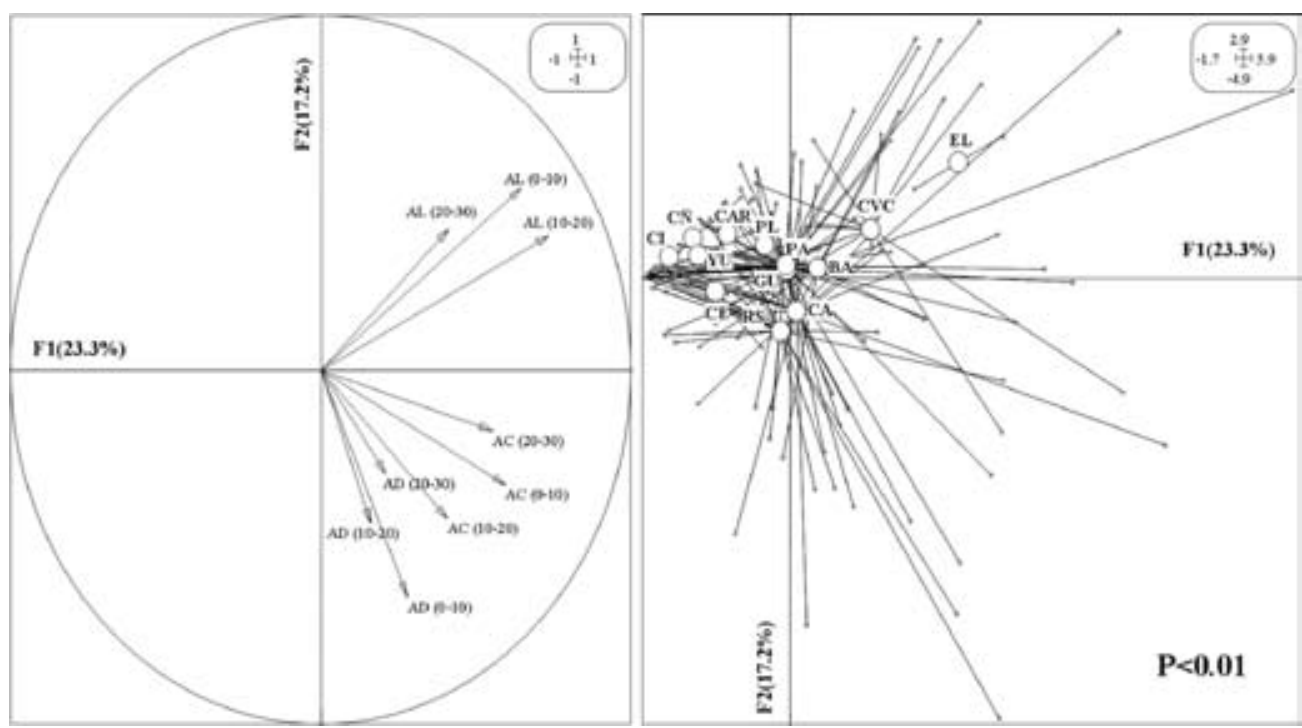

Figura 4. Proyección de los patrones asociados con la conservación y degradación de los usos del terreno en la cuenca del río de La Vieja, para las variables biológicas ( $\mathrm{AL}=$ abundancia de lombrices, $\mathrm{AC}=$ abundancia de coleópteros y $\mathrm{AD}=$ abundancia de diplópodos $)$, en las diferentes profundidades del suelo (0-10, 10-20 y 20-30 cm), definidas por el ACP en el espacio factorial. Para las siglas de los usos, ver leyenda de la Fig. 3.

El análisis de coinercia mostró que las variables fisicoquímicas y biológicas explicaron el $95.1 \%$ de la variabilidad total $(\mathrm{p}<0.02)$. El factor $1(88.3 \%)$ mostró alta correlación entre las variables humedad gravimétrica en $0-30 \mathrm{~cm}$ de profundidad y abundantes coleópteros en 0-20 $\mathrm{cm}$ y diplópodos en 0-10 $\mathrm{cm}$, y opuso la alta densidad aparente en 0-30 cm y altas abundancias de diplópodos en 10-20 y en lombrices de $20-30 \mathrm{~cm}$. El factor $2(6.8 \%)$ contrapuso la altitud del local y la abundancia de lombrices en $0-10 \mathrm{~cm}$ (Fig. 5).

\section{DISCUSIÓN}

El esquema metodológico utilizado ( 9 ventanas de $1 \mathrm{~km}^{2}, 31$ fincas, 16 puntos muestreados con TSBF, 13 usos del terreno y 12 variables) en los muestreos y el análisis estadístico riguroso (análisis de varianza, $\mathrm{ACP}$, análisis discriminante y de coinercia) de la información permitieron hibridar de manera interdisciplinaria los registros provenientes de diferentes propiedades del suelo y de los agroecosistemas en la cuenca del río La Vieja, para generar patrones de uso del terreno y discriminar diferentes tipos de uso de acuerdo con los cambios en los arreglos que introducen los agricultores. 


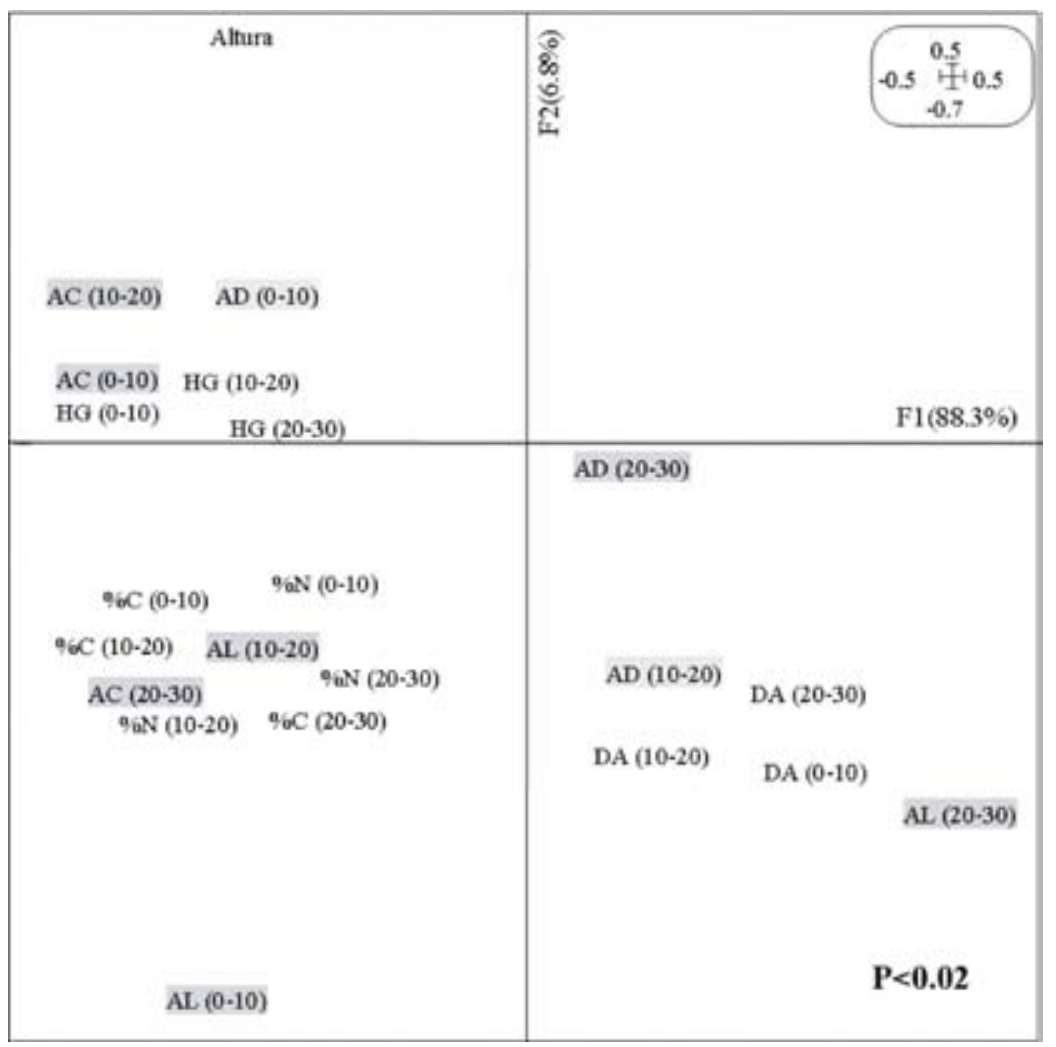

Figura 5. Análisis de coinercia a tres profundidades del suelo entre variables fisicoquímicas y biológicas. Para las siglas de los usos y de las variables, ver leyendas de las Fig. 3 y 4.

Las características fisicoquímicas del suelo delinearon cuatro patrones asociados con el tipo de uso del terreno: sitios con alta humedad del suelo (CE, RS y BA) opuestos al de mayor densidad del suelo (PL, YU, CI) y sitios con altos valores de C y N (CAR, EL, CA, CVC) opuestos a los de bajos valores (CÑ, PA y GU). Con los atributos biológicos se trazaron tres patrones de usos: sitios con alta abundancia de lombrices y baja de coleópteros y diplópodos (CVC y EL) opuestos a sitios con baja abundancia de los mismos (CI, YU, CÑ, CE) y usos con valores intermedios de abundancia y presencia de lombrices, coleópteros y diplópodos (RS, GU, CA, PL, BA, PA).

El análisis de coinercia al combinar variables fisicoquímicas por biológicas generó tres patrones. El primero incluyó usos con alta humedad gravimétrica y abundancia de coleópteros y diplópodos, favorecidos por el aporte y calidad nutricional de la hojarasca de árboles de Sauravia scabra, Miconia aeruginosa, Cupania americana, 
Piper sp., Brosimum utile, Ocotea insulares. El segundo patrón reunió suelos de alta densidad aparente y abundantes en lombrices y diplópodos en 20-30 cm cubiertos por pastizales y cítricos que alojaron lombrices endogeicas (Polypheretima elongata y Pontoscolex corethrurus), que soportan perturbaciones por labranza, aplicaciones de agroquímicos y suelos compactos (Vertisoles, Alfisoles y Mollisoles) (Huerta et al. 2006). El tercer patrón aglutinó suelos con altos contenidos de $\mathrm{C}, \mathrm{N}$ y abundancias de lombrices 0-20 cm y coleópteros en 20-30 cm, cubiertos por agroecosistemas con plantas de variada arquitectura radicular y de biomasa aérea (cafetal con sombrío con las especies Coffea arabica, Cestrum sp. aff microcalix, Piper sp., Meriania speciosa) y relicto de selva (Sauravia scabra, Miconia aeruginosa, Cupania americana, Piper sp., Brosimum utile, Ocotea insularis), que favorecieron la mezcla de algunas especies de lombrices nativas y exóticas tales como Martiodrilus (Maipure) murindo, M. (Mai.) agricola, Dichogaster medellina, Glossodrilus griseus, Amynthas gracilis, Perionyx excavatus y Dichogaster annae.

El análisis de información procedente de la asociación entre usos del terreno, itinerarios técnicos de manejo y variables fisicoquímicas y biológicas ayuda a diferenciar ambientes hostiles o amigables para la diversidad en macroinvertebrados edáficos. La asignación de terrenos y el sistema de manejo incide en la generación de ambientes o nichos que favorecen o limitan la proliferación de los individuos en los agroecosistemas. En este caso, la introducción de prácticas o técnicas culturales (tala de selva subandina, quemas, cobertura de terrenos con vegetación permanente o transitoria de los agroecosistemas, cultivos en surco continuo con aplicación de agroquímicos, introducción de pastizales africanos) interrumpe fuertemente la conectividad de los sistemas para los procesos de desplazamiento y recolonización, modificando los mapas de los paisajes. La evaluación en cada ventana de las coberturas sembradas o usos del terreno evidenció los efectos de los sistemas de manejo de los agroecosistemas sobre las características fisicoquímicas y biológicas del sitio asociadas con la intervención humana.

Se intentó explicar la riqueza y composición de los ensamblajes de la edafofauna por hábitats particulares construidos por las intervenciones antrópicas desde atributos culturales definidos por los procesos de innovación en cada localidad. Se espera avanzar en la diferenciación de "estructuras claves", elementos del paisaje que proporcionan recursos cruciales para muchas especies y que estimulan la diversidad (Bennet et al. 2006). La alta heterogeneidad del paisaje en parches de espacios irregulares y la diferencia en niveles de intensificación antropogénica, permiten calificar el paisaje de la subregión como producto dinámico de la interacción entre tradiciones e innovaciones culturales.

La herramienta utilizada en el análisis de la información permitió involucrar diferentes variables disciplinarias para ir avanzando en representaciones sintéticas del paisaje con uso potencial en la definición de áreas amigables o críticas para la agro- 
biodiversidad, elaboración de mapas de riesgo según prácticas o técnicas de manejo, evaluación de sistemas de uso del terreno en las funciones de conservación o la generación de políticas de protección para el manejo de los recursos y la biodiversidad (Velásquez et al. 2007).

Además se demostró significativamente la sensibilidad de atributos tales como composición y estructura de lombrices, coleópteros y diplópodos que unidas a algunas variables fisicoquímicas mostraron tres patrones del uso del terreno, lo cual se convierte en una herramienta de planificación importante para detectar umbrales de uso en los agroecosistemas, espacios hostiles o amigables para la fauna y, enseño opciones para agrupar las irregularidades funcionales y de estructura en el paisaje.

\section{AGRADECIMIENTOS}

Los autores miembros del grupo Gestión en Agroecosistemas Tropicales Andinos (GATA) de la Universidad Tecnológica de Pereira agradecen a los agricultores criadores de la cuenca del río La Vieja por haber facilitado los predios para la realización del estudio y también a la Universidad Tecnológica de Pereira, al Centro de Investigaciones en Biodiversidad y Recursos Genéticos (CIEBREG) y a Colciencias por la financiación del proyecto "Valoración de los bienes y servicios de la biodiversidad para el desarrollo sostenible de paisajes rurales colombianos, Complejo Ecorregional de los Andes del Norte (CEAN)".

\section{LITERATURA CITADA}

Anderson, J. M. \& J. S. I. Ingram. 1993. Tropical soil biology and fertility: A handbook of methods, $2^{\text {nd }}$ Ed. CAB International, Oxford.

Bennet, A. F., J. Q. Radford \& A. Haslem. 2006. Properties of land mosaics: implications for nature conservation in agricultural environments. Biological Conservation. 133: 250-264.

BIOASSESS. 2003. Biodiversity assessment manual. Version 1- Assessment protocols. http://www. nbu.ac.uk/bioassess/.

Blake, G. R. \& K. H. Hartge, 1986. Bulk density. Pp. 363-382. In: A. Klute (Ed). Methods of soil analysis. Part 1. Physical and mineralogical methods, $2^{\text {nd }}$ ed. Agronomy Monographs No. 9. SSSA, Madison.

Blair, J. M., P. J. Bohlen, \& D. W. Freckman, 1996. Soil invertebrates as indicators of soil quality. Pp. 273-291. In: J.W. Doran and A.J. Jones (Eds). Methods for assessing soil quality. SSSA Special Publication No. 16. SSSA, Madison.

Capra, F. 1998. La trama de la vida: Una nueva perspectiva de los sistemas vivos. Editorial Anagrama, Barcelona.

Cochran, W. G. 1974. Técnicas de muestreo. Cuarta Edición. Continental S.A., México.

Feijoo, A. \& E. B. Knapp, 1998a. El papel de los macroinvertebrados como indicadores de fertilidad y perturbación de suelos de Laderas. Suelos Ecuatoriales. 28: 254-259.

Feijoo, A. \& H. Quintero. 1998b. Glossoscolecidae de una región de los Andes del Departamento del Valle, Colombia. Suelos Ecuatoriales. 28: 249-253.

Feijoo, A., E. B. Knapp, P. Lavelle \& A. Moreno. 1999. Quantifying soil macrofauna in a Colombian watershed. Pedobiologia. 43: 513-517. 
Feijoo, M. A., H. Quintero \& C. E. Fragoso. 2006. Earthworm communities in forest and pastures of the Colombian Andes. Caribbean Journal of Science. 42: 301-310.

Hamblin, W. K. \& E. H. Christiansen, 1998. Earth's dynamic systems, 8th edition. Prentice Hall, Upper Saddle River.

Huerta, E., C. Fragoso, J. Rodríguez-Olan, I. Evia-Castillo, E. Montejo-Meneses, E. M. De La Cruz-Mondragon \& R. García-Hernández. 2006. Presence of exotic and native earthworms in principal agro and natural systems in Central and South-Eastern Tabasco, Mexico. Caribbean Journal of Science. 42: 359-365.

Lavelle, P., D. Bignell, M. Legage, V. Volters, P. Roger, P. Ineson, B. Heal \& S. Dhillion. 1997. Soil function in a changing world: the role of soil ecosystem engineers. European Journal of Soil Biology. 33: 159-193.

Lavelle, P., T. Decaëns, M. Aubert, S. Barot, M. Blouin, F. Bureau, P. Margarie, P. Mora \& J.-P. Rossi. 2006. Soil invertebrates and ecosystem services. European Journal of Soil Biology. 42: 3-15.

MAVDT, IDEAM, CRQ, CVC, CARDER \& GTZ. 2005. Proyecto piloto "Ordenamiento de la Cuenca del río La Vieja". Bogotá.

McLachlan, G. J. 1992. Discriminant analysis and statistical pattern recognition. Wiley Press, New York.

Mathieu, J., J. P. Rossi, M. Grimaldi, P. Mora, P. Lavelle \& C. Rouland. 2005. A multi-scale study of soil macrofauna biodiversity in Amazonian pastures. Biology and Fertility of Soils. 40: 300-305.

Parr, J. F., S. B. Papendick, S. B. Hornick \& R. E. Meyer. 1992. Soil quality: Attributes and relationships to alternative and sustainable agriculture. American Journal of Alternative Agriculture. 7: 5-11

Siegel, S. 1974. Estadística no paramétrica aplicada a las ciencias de la conducta, Segunda edición. Editorial Trillas, México.

SPSS (Statistical Product and Service Solution) Inc., 1999.

Thioulouse, J., D. Chessel, S. Dolédec \& J. M. Olivier. 1997. ADE-4: A multivariate analysis and graphical display software. Statistical Computation. 7: 2-15.

Velasquez, E., P. Lavelle \& M. Andrade. 2007. GISQ, a multifunctional indicator of soil quality. Soil Biology and Biochemistry. 39:3066-3080.

Vincent, P. J., J. M. Haworth, J. G. Griffiths \& R. Collins. 1976. The detection of randomness in plant patterns. Journal of Biogeography. 3: 373-380.

Zúñiga, M. C., H. Quintero \& A. Feijoo. 2003. Trayectoria de los sistemas de cría en un área del piedemonte de Alcalá, Valle del Cauca. Scientia et Tecnica. 9: 81-86.

Zúñiga, M. C., A. Feijoo \& H. Quintero. 2004. Diseño de una propuesta metodológica para interpretar el huerto habitacional en un área del Valle del Cauca. Scientia et Tecnica. 10: 291-296. 